\title{
On the irreversibility of fluctuations
}

\author{
A.G.Sitenko \\ Bogolyubov Institute for Theoretical Physics \\ 03143 Kyiv, Ukraine
}

Received March 6, 2000

Interrelationship of classical and quantum fluctuations is discussed. With energy dissipation being taken into account, fluctuations are shown to be irreversible even in the case of classical systems. An analogy to Kubo relation for classical fluctuations is derived. Fluctuations in both equilibrium and nonequilibrium (though stationary) states of the system are considered.

Key words: classical and quantum fluctuations, irreversibility of fluctuations, Kubo formula

PACS: $05.40,42.50 . L, 05.70 . L, 52.25 . G$

\section{Introduction}

Basic theory of fluctuations of physical quantities in both classical and quantum systems is discussed in the brilliant books $[1,2]$. In particular, these books give a detailed treatment of electromagnetic fluctuations in equilibrium systems. The authors of $[1,2]$ introduce symmetrized binary correlation functions which are invariant with respect to time reversal and employ this approach for a detailed consideration of both classical and quantum fluctuations. However, they do not pay enough attention to the question whether fluctuations are time-reversible or time-irreversible. The pair correlation function for the fluctuations of classical quantities is assumed to be symmetric with respect to time reversal (see equation (118.3) of [2]) and hence fluctuations in classical systems are treated as time-reversible. This statement contradicts the well known Kubo relation [3] which determines the relation of the linear response of the system to external perturbations and the antisymmetrized pair correlation function. Kubo relation for quantum fluctuations is derived in [2] (equation (126.8) of [2]); it suggests an irreversible nature of quantum fluctuations.

Irreversibility of fluctuations even in the case of classical systems and applicability of Kubo relation to the description of classical fluctuations are discussed in this paper. Moreover, the consideration is extended to the case of nonequilibrium stationary systems. 


\section{Correlation functions}

We employ the notation introduced in [2] and consider time fluctuations of the quantity $x(t)$. We assume this quantity to be real and such that its mean value is equal to zero in the absence of external perturbations. We introduce the relevant Hermitian operator $\hat{x}(t)$ for this quantity and employ the Fourier transformation, i.e.,

$$
\hat{x}(t)=\frac{1}{2 \pi} \int_{-\infty}^{\infty} \mathrm{d} \omega \mathrm{e}^{-\mathrm{i} \omega t} \hat{x}_{\omega}, \quad \hat{x}_{\omega}=\int_{-\infty}^{\infty} \mathrm{d} t \mathrm{e}^{\mathrm{i} \omega t} \hat{x}(t) .
$$

If the state of the system is stationary, then the correlation of fluctuations of this quantity taken at different time instants, say 0 and $t$, depends only on the time interval between these instants. We define the correlation functions

$$
\langle x(0) x(t)\rangle \equiv\left\langle x^{2}\right\rangle_{t}, \quad\langle x(t) x(0)\rangle \equiv\left\langle x^{2}\right\rangle_{-t},
$$

where angular brackets in the left-hand parts of the equations denote quantum and statistical averaging. The relevant spectral distributions of fluctuations, $\left\langle x^{2}\right\rangle_{\omega}$ and $\left\langle x^{2}\right\rangle_{-\omega}$, are determined by the Fourier transforms of the correlation functions (2). We note that the average product of Fourier components of fluctuations $\hat{x}_{\omega}^{\dagger}$ and $\hat{x}_{\omega^{\prime}}$ is related to the spectral distribution of fluctuations as given by

$$
\left\langle\hat{x}_{\omega}^{\dagger} \hat{x}_{\omega^{\prime}}\right\rangle=2 \pi \delta\left(\omega-\omega^{\prime}\right)\left\langle x^{2}\right\rangle_{\omega}
$$

(the symbol $\dagger$ implies the Hermitian conjugation operation).

We calculate the mean value of the operators product $\hat{x}_{\omega}^{\dagger} \hat{x}_{\omega^{\prime}}$ for some quantum state $n$ with energy $E_{n}$ and average the expression obtained over the statistical distribution of quantum states $f\left(E_{n}\right)$. Thus we obtain the spectral distributions of fluctuations (2) in the form

$$
\begin{aligned}
\left\langle x^{2}\right\rangle_{\omega} & =2 \pi \sum_{m, n} f\left(E_{n}\right)\left|x_{n m}\right|^{2} \delta\left(\omega-\omega_{n m}\right), \\
\left\langle x^{2}\right\rangle_{-\omega} & =2 \pi \sum_{m, n} f\left(E_{n}\right)\left|x_{n m}\right|^{2} \delta\left(\omega+\omega_{n m}\right),
\end{aligned}
$$

where $x_{n m}$ is the matrix element of the operator $\hat{x}$ and $\omega_{n m}=\left(E_{n}-E_{m}\right) / \hbar$ is the frequency of the transition between the states $n$ and $m$.

The quantity $\left\langle x^{2}\right\rangle_{-\omega}$ determines the spectral distribution of fluctuations reversed in time with respect to fluctuations described by the spectral distribution $\left\langle x^{2}\right\rangle_{\omega}$. We rename $n \leftrightarrow m$ in (5) and employ the properties of the delta-function. Thus the spectral distribution (5) takes the form

$$
\left\langle x^{2}\right\rangle_{-\omega}=2 \pi \sum_{m, n} f\left(E_{n}-\hbar \omega\right)\left|x_{n m}\right|^{2} \delta\left(\omega-\omega_{n m}\right) .
$$

If the system is in thermodynamic equilibrium, then the function of statistical distribution of states, $f\left(E_{n}\right)$, should be taken in the Gibbs form, i.e.,

$$
f\left(E_{n}\right)=\mathrm{e}^{\frac{\mathcal{F}-E_{n}}{T}},
$$


where $E_{n}$ is the energy of nth level, $\mathcal{F}$ is the free energy, and $T$ is the temperature of the system. Comparing (6) to (4) yields a relation for the spectral distributions of unreversed and reversed fluctuations, i.e.,

$$
\left\langle x^{2}\right\rangle_{-\omega}=\mathrm{e}^{\frac{\hbar \omega}{T}}\left\langle x^{2}\right\rangle_{\omega}
$$

\section{Energy dissipation and relationship with the correlation of fluctuations}

Correlation functions (4) and (6) can be related to the energy dissipated in the system exposed to an external perturbation. If the perturbation is periodic with the frequency $\omega$ and its energy is proportional to the quantity $x$, i.e.,

$$
\hat{V}=-f(t) \hat{x}, \quad f(t)=\frac{1}{2}\left(f_{0} \mathrm{e}^{-\mathrm{i} \omega t}+f_{0}^{*} \mathrm{e}^{\mathrm{i} \omega t}\right),
$$

where $f_{0}$ is constant, then the mean energy absorbed by the system per unit time is given by

$$
Q=\frac{\omega}{4 \hbar}\left|f_{0}\right|^{2}\left\{\left\langle x^{2}\right\rangle_{-\omega}-\left\langle x^{2}\right\rangle_{\omega}\right\} .
$$

With the external perturbation (8) being present, the mean value $\langle x(t)\rangle$ is not equal to zero; it is proportional to the perturbation $f(t)$, i.e.,

$$
\langle x(t)\rangle=\int_{0}^{\infty} \mathrm{d} \tau \alpha(\tau) f(t-\tau),
$$

where $\alpha(\tau)$ is the linear response of the system. According to the causality principle, integration in (10) extends over time previous to $t$. Having performed the Fourier transformation, we find that

$$
\langle x\rangle_{\omega}=\alpha(\omega) f_{\omega}
$$

where

$$
\alpha(\omega)=\int_{0}^{\infty} \mathrm{d} t \mathrm{e}^{\mathrm{i} \omega t} \alpha(t) .
$$

The linear response $\alpha(\omega)$ is an analytic function of the frequency $\omega$.

The energy absorbed by the system is immediately expressed in terms of the linear response, we have

$$
Q=\frac{\omega}{2}\left|f_{0}\right|^{2} \alpha^{\prime \prime}(\omega)
$$

where $\alpha^{\prime \prime}(\omega)$ is the imaginary part of the coefficient $\alpha(\omega)$. Comparing this expression to (9) yields the basic relation of the fluctuation theory, i.e.,

$$
\alpha^{\prime \prime}(\omega)=\frac{1}{2 \hbar}\left\{\left\langle x^{2}\right\rangle_{-\omega}-\left\langle x^{2}\right\rangle_{\omega}\right\}
$$


which relates the spectral distributions of time-reversed fluctuations with the dissipative properties of the system.

We introduce the symmetrized correlation function

$$
\left\langle x^{2}\right\rangle_{\omega}^{\mathrm{s}}=\frac{1}{2}\left\{\left\langle x^{2}\right\rangle_{\omega}+\left\langle x^{2}\right\rangle_{-\omega}\right\}
$$

and make use of relation (7). Thus, directly from (13), we obtain the fluctuationdissipation relation for an equilibrium system [4], i.e.,

$$
\left\langle x^{2}\right\rangle_{\omega}^{\mathrm{s}}=\hbar \operatorname{cth} \frac{\hbar \omega}{2 T} \alpha^{\prime \prime}(\omega) .
$$

In a similar manner we find that spectral distributions of fluctuations (9) and (6) for the equilibrium state of the system are given by

$$
\left\langle x^{2}\right\rangle_{\omega}=\left\langle x^{2}\right\rangle_{\omega}^{\mathrm{s}}-\hbar \alpha^{\prime \prime}(\omega), \quad\left\langle x^{2}\right\rangle_{-\omega}=\left\langle x^{2}\right\rangle_{\omega}^{\mathrm{s}}+\hbar \alpha^{\prime \prime}(\omega) \text {. }
$$

\section{Kubo relation and irreversibility of fluctuations}

Since the linear response $\alpha(\omega)$ is an analytic function without singularities in the upper halfplane of complex frequency $\omega[2]$, we have

$$
\frac{1}{\pi} \int_{-\infty}^{\infty} \mathrm{d} \omega^{\prime} \frac{\alpha^{\prime \prime}\left(\omega^{\prime}\right)}{\omega^{\prime}-\omega-\mathrm{i} 0}=\alpha(\omega)-\alpha(\infty)
$$

Within the context of the relation

$$
\frac{1}{\pi} \int_{-\infty}^{\infty} \mathrm{d} \omega^{\prime} \frac{\left\langle x^{2}\right\rangle_{-\omega^{\prime}}-\left\langle x^{2}\right\rangle_{\omega^{\prime}}}{\omega^{\prime}-\omega-i 0}=2 \mathrm{i} \int_{0}^{\infty} \mathrm{d} t \mathrm{e}^{\mathrm{i} \omega t}\left\{\left\langle x^{2}\right\rangle_{-t}-\left\langle x^{2}\right\rangle_{t}\right\}
$$

we obtain from (13) the Kubo formula [3], i.e.,

$$
\alpha(\omega)-\alpha(\infty)=\frac{\mathrm{i}}{\hbar} \int_{0}^{\infty} \mathrm{d} t \mathrm{e}^{\mathrm{i} \omega t}\{\langle x(t) x(0)\rangle-\langle x(0) x(t)\rangle\} .
$$

By definition, $\left\langle x^{2}\right\rangle_{\omega}$ and $\left\langle x^{2}\right\rangle_{-\omega}$ are spectral distributions of fluctuations reversed in time. According to (13), their difference is determined by the linear response imaginary part $\alpha^{\prime \prime}(\omega)$ which characterizes the energy dissipation in the system. The observation that spectral distributions $\left\langle x^{2}\right\rangle_{\omega}$ and $\left\langle x^{2}\right\rangle_{-\omega}$ are not equal implies that fluctuations in a dissipative system are irreversible.

\section{Irreversibility of classical fluctuations and classical analogy to Kubo formula}

The above conclusion concerning the time-irreversibility of fluctuations has been formally derived in terms of quantum mechanics. Meanwhile, fluctuations are irreversible in classical systems as well. Indeed, though for $\hbar \rightarrow 0$ (i.e., for a classical 
system) spectral distributions of time-reversed fluctuations,

$$
\left\langle x^{2}\right\rangle_{\omega}^{\mathrm{cl}}=\lim _{\hbar \rightarrow 0}\left\langle x^{2}\right\rangle_{\omega}, \quad \text { and } \quad\left\langle x^{2}\right\rangle_{-\omega}^{\mathrm{cl}}=\lim _{\hbar \rightarrow 0}\left\langle x^{2}\right\rangle_{-\omega}
$$

are equal within the context of (4) and (6), their difference nevertheless determines the imaginary part of the linear response for the classical system. In the limiting case $\hbar \rightarrow 0$, equation (6) yields

$$
\left\langle x^{2}\right\rangle_{-\omega} \underset{\hbar \ll 1}{\simeq}\left\langle x^{2}\right\rangle_{\omega}-\hbar \omega \frac{\partial}{\partial E}\left\langle x^{2}\right\rangle_{\omega}
$$

where $\partial\left\langle x^{2}\right\rangle_{\omega} / \partial E$ is the correlation function averaged over the derivative of the distribution function,

$$
\frac{\partial}{\partial E}\left\langle x^{2}\right\rangle_{\omega} \equiv 2 \pi \sum_{m, n} \frac{\partial f\left(E_{n}\right)}{\partial E_{n}}\left|x_{m n}\right|^{2} \delta\left(\omega-\omega_{n m}\right)
$$

Thus, in the case of classical fluctuations we have

$$
\frac{1}{2} \lim _{\hbar \rightarrow 0} \frac{\left\langle x^{2}\right\rangle_{-\omega}-\left\langle x^{2}\right\rangle_{\omega}}{\hbar}=-\frac{\omega}{2} \frac{\partial}{\partial E}\left\langle x^{2}\right\rangle_{\omega},
$$

and hence the condition of fluctuation irreversibility reduces to

$$
\alpha^{\prime \prime}(\omega)=-\frac{\omega}{2} \frac{\partial}{\partial E}\left\langle x^{2}\right\rangle_{\omega} .
$$

Since the linear response is analytical, we obtain from (20)

$$
\alpha(\omega)-\alpha(\infty)=-\frac{1}{2 \pi} \int_{-\infty}^{\infty} \mathrm{d} \omega^{\prime} \frac{\omega^{\prime} \frac{\partial}{\partial E}\left\langle x^{2}\right\rangle_{\omega^{\prime}}}{\omega^{\prime}-\omega-\mathrm{i} 0}
$$

which implies that the linear response is determined by the time-irreversible part of the correlation function. If the system is in the state of thermodynamic equilibrium, then we have

$$
\frac{\partial}{\partial E}\left\langle x^{2}\right\rangle_{\omega}=-\frac{1}{T}\left\langle x^{2}\right\rangle_{\omega}^{\mathrm{s}}
$$

We substitute the latter expression in (21) and carry out integration over the frequency $\omega^{\prime}$. Thus we obtain a classical analogy to Kubo formula for an equilibrium system. It is given by

$$
\alpha(\omega)-\alpha(\infty)=-\frac{1}{2 T} \int_{0}^{\infty} \mathrm{d} t \mathrm{e}^{\mathrm{i} \omega t} \frac{\partial}{\partial t}\{\langle x(t) x(0)\rangle+\langle x(0) x(t)\rangle\}
$$




\section{Inversion of the fluctuation-dissipation relation}

It is clear that in order to calculate the linear response from (21), taking into account the interaction in the system in the linear approximation, it is sufficient to substitute the correlation function in the right-hand part disregarding the selfconsistent interaction. Thus, in the linear approximation we have

$$
\alpha(\omega)-\alpha(\infty)=-\frac{1}{2 \pi} \int_{-\infty}^{\infty} \mathrm{d} \omega^{\prime} \frac{\omega^{\prime} \frac{\partial}{\partial E}\left\langle x^{2}\right\rangle_{\omega^{\prime}}^{0}}{\omega^{\prime}-\omega-\mathrm{i} 0}
$$

where $\frac{\partial}{\partial E}\left\langle x^{2}\right\rangle_{\omega}^{0}$ is the correlation function of the system without interaction. For an equilibrium system, in particular, we have

$$
\alpha(\omega)-\alpha(\infty)=\frac{1}{2 \pi} \frac{1}{T} \int_{-\infty}^{\infty} \mathrm{d} \omega^{\prime} \frac{\omega^{\prime}\left\langle x^{2}\right\rangle_{\omega^{\prime}}^{0}}{\omega^{\prime}-\omega-\mathrm{i} 0} .
$$

Relations (23) and (24) can be treated as an inversion of the fluctuation-dissipation relation $[5,6]$.

\section{Electromagnetic fluctuations}

Now we extend the consideration to the case of fluctuations of spatially distributed quantities. For example we consider electromagnetic fluctuations in a medium, in particular, fluctuations of the current density $\mathbf{j}(\mathbf{r}, t)$. We assume the system to be spatially homogeneous and stationary and put the average current to be equal to zero, $\langle\mathbf{j}(\mathbf{r}, t)\rangle=0$.

We introduce the space-time Fourier transformation for the current fluctuations,

$$
\mathbf{j}_{\mathbf{k} \omega}=\int_{-\infty}^{\infty} \mathrm{d} t \mathrm{e}^{\mathrm{i} \omega t} \mathbf{j}_{\mathbf{k}}(t), \quad \mathbf{j}_{\mathbf{k}}(t)=\int \mathrm{d} \mathbf{r} \mathrm{e}^{-\mathrm{i} \mathbf{k r}} \mathbf{j}(\mathbf{r}, t),
$$

and calculate the average product of fluctuation components

$$
\left\langle j_{i}^{\dagger}(\mathbf{k}, \omega) j_{j}\left(\mathbf{k}^{\prime}, \omega^{\prime}\right)\right\rangle \equiv(2 \pi)^{4} \delta\left(\omega-\omega^{\prime}\right) \delta\left(\mathbf{k}-\mathbf{k}^{\prime}\right)\left\langle j_{i} j_{j}\right\rangle_{\mathbf{k} \omega},
$$

Thus we obtain an expression for the spectral distribution of fluctuations given by

$$
\left\langle j_{i} j_{j}\right\rangle_{\mathbf{k} \omega}=2 \pi \sum_{m, n} f\left(E_{n}\right) j_{i}(\mathbf{k})_{m n}^{*} j_{j}(\mathbf{k})_{m n} \delta\left(\omega-\omega_{n m}\right),
$$

where $j_{i}(\mathbf{k})_{m n}$ is the matrix element of the operator $j_{\mathbf{k}}(t)$ for the transition from $n$ to $m$. We note that

$$
\mathbf{j}^{\dagger}(\mathbf{k})_{n m} \equiv \mathbf{j}(\mathbf{k})_{m n}^{*}=\mathbf{j}(-\mathbf{k})_{n m}
$$

The spectral distribution of fluctuations reversed in time with respect to the fluctuations described by the distribution (27) is given by

$$
\left\langle j_{i} j_{j}\right\rangle_{-\mathbf{k}-\omega}=2 \pi \sum_{m, n} f\left(E_{n}-\hbar \omega\right) j_{i}(\mathbf{k})_{m n} j_{j}(\mathbf{k})_{m n}^{*} \delta\left(\omega-\omega_{n m}\right) .
$$


It is not difficult to verify that

$$
\left\langle j_{i} j_{j}\right\rangle_{-\mathbf{k}-\omega}^{*}=\left\langle j_{j} j_{i}\right\rangle_{-\mathbf{k}-\omega} .
$$

For the equilibrium state we have

$$
\left\langle j_{j} j_{i}\right\rangle_{-\mathbf{k}-\omega}=\mathrm{e}^{\frac{\hbar \omega}{T}}\left\langle j_{i} j_{j}\right\rangle_{\mathbf{k} \omega} .
$$

We take the perturbation energy to be described by the expression

$$
\hat{V}=-\int \mathrm{d} \mathbf{r} \mathbf{A}(\mathbf{r}, t) \mathbf{j}(\mathbf{r}, t)
$$

where $\mathbf{A}(\mathbf{r}, t)$ is a potential with harmonic time dependence, i.e.,

$$
\mathbf{A}(\mathbf{r}, t)=\frac{1}{2} \sum_{\mathbf{k}}\left\{\mathbf{A}_{\mathbf{k} \omega} \mathrm{e}^{\mathrm{i} \mathbf{k r}-\mathrm{i} \omega t}+\mathbf{A}_{\mathbf{k} \omega}^{*} \mathrm{e}^{-\mathrm{i} \mathbf{k r}+\mathrm{i} \omega t}\right\} .
$$

The external perturbation (32) gives rise to a current $\mathbf{j}(\mathbf{r}, t)$ with nonzero mean value proportional to the potential $\mathbf{A}(\mathbf{r}, t)$. The linear relation for these quantities may be written as

$$
j_{i}(\mathbf{k}, \omega)=\alpha_{i j}(\omega, \mathbf{k}) A_{j}(\mathbf{k}, \omega),
$$

where $\alpha_{i j}(\omega, \mathbf{k})$ are macroscopic coefficients which determine the dissipative properties of the system.

The mean energy absorbed by the system, on the one hand is expressed in terms of the correlation functions (27) and (29), and on the other hand it is determined by the dissipative linear response. We equate the relevant expressions and thus obtain the basic relation of the theory of electromagnetic fluctuations, i.e.,

$$
\frac{1}{\hbar}\left\{\left\langle j_{i} j_{j}\right\rangle_{-\mathbf{k}-\omega}^{*}-\left\langle j_{i} j_{j}\right\rangle_{\mathbf{k} \omega}\right\}=\mathrm{i}\left\{\alpha_{i j}^{*}(\omega, \mathbf{k})-\alpha_{j i}(\omega, \mathbf{k})\right\} .
$$

For an isotropic medium, the coefficients $\alpha_{i j}(\omega, \mathbf{k})$ are symmetric with respect to the indices $i$ and $j$,

$$
\alpha_{i j}(\omega, \mathbf{k})=\alpha_{j i}(\omega, \mathbf{k})
$$

In this case the difference of spectral distributions of time-reversed electromagnetic fluctuations is directly determined by the imaginary part of the dissipative coefficient $\alpha_{i j}(\omega, \mathbf{k})$. We have

$$
\frac{1}{2 \hbar}\left\{\left\langle j_{i} j_{j}\right\rangle_{-\mathbf{k}-\omega}^{*}-\left\langle j_{i} j_{j}\right\rangle_{\mathbf{k} \omega}\right\}=\alpha_{i j}^{\prime \prime}(\omega, \mathbf{k})
$$

Making use of (30) and (31), we obtain from (34) or (35) the fluctuation-dissipation relation for the symmetrized correlation function for the case of equilibrium system $[6]$.

For classical systems (in the limiting case $\hbar \rightarrow 0$ ), the relation of fluctuation irreversibility (34) reduces to the form

$$
-\omega \frac{\partial}{\partial E}\left\langle j_{i} j_{j}\right\rangle_{\mathbf{k} \omega}=\mathrm{i}\left\{\alpha_{i j}^{*}(\omega, \mathbf{k})-\alpha_{j i}(\omega, \mathbf{k})\right\}
$$

where $\frac{\partial}{\partial E}\left\langle j_{i} j_{j}\right\rangle_{\mathbf{k} \omega}$ is the correlation function (27) averaged over the derivative of the distribution function. The relevant Kubo relation or the inverse fluctuationdissipation relation follow immediately from (34) or (36). 


\section{References}

1. Landau L.D., Lifshitz E.M. Electrodynamics of Continuous Media. Moscow, GITTL, 1957 (in Russian).

2. Landau L.D., Lifshitz E.M. Statistical Physics. Pergamon Press, Oxford, 1969.

3. Kubo R. Statistical-mechanical theory of irreversible processes. // Journ. Phys. Society of Japan, 1957, vol. 12, p. 570-586.

4. Callen H., Welton T. Irreversibility and generalized noise. // Phys. Rev., 1951, vol. 83, p. 34-46.

5. Sitenko A.G. Fluctuation-dissipative ratio for non-equilibrium systems. // Ukr. Journ. Phys., 1966, vol. 11, p. 1161-1166.

6. Sitenko A.G. Electromagnetic Fluctuations in Plasma. Academic Press, New York, 1967.

\section{Про незворотність флюктуацій у часі}

\section{О.Г.Ситенко}

Інститут теоретичної фізики ім. М.М. Боголюбова НАН України, 03143 Київ, вул. Метрологічна, 146

Отримано 6 березня 2000 р.

Обговорюється взаємозв'язок між класичними та квантовими флюктуаціями. Вказано на незворотність (навіть у випадку класичних систем) флюктуацій за наявності дисипації енергії. Одержано аналог співвідношення Кубо для класичних флюктуацій. Розглянуто флюктуації як у рівноважних, так і в нерівноважних (але стаціонарних) станах системи.

Ключові слова: класичні і квантові флюктуації, незворотність флюктуацій, формула Кубо

PACS: $05.40,42.50 . L, 05.70 . L, 52.25 . G$ 